\title{
Laser Desorption Postionization Mass Spectrometry and X-ray Surface Scattering for the Analysis of Bacterial Biofilms
}

\author{
P.D. Edirisinghe, ${ }^{*}$ C.A. Crot, ${ }^{*}$ J.F. Moore, ${ }^{* *}$ W.F. Calaway, ${ }^{* *}$ M.J. Pellin, ${ }^{* * *}$ D.G. Schultz, ${ }^{*}$ \\ K.A. Skinner-Nemec, ${ }^{* * * *}$ C.S. Giometti, ${ }^{* * * *}$ A. Kilislioglu, ${ }^{* * * * *}$ and L. Hanley* \\ * Department of Chemistry, University of Illinois at Chicago, Chicago, IL 60607-7061 \\ ** Mass Think, 2308 Hartford Ct., Naperville, IL 60565-3123 \\ *** Materials Science Division, Argonne National Laboratory, 9700 S Cass Ave, Argonne, IL \\ 60439 \\ **** Biosciences Division, Argonne National Laboratory, 9700 S Cass Ave, Argonne, IL 60439 \\ ***** Istanbul University, Department of Chemistry, Avcilar 34320, Istanbul, Turkey
}

Microbial biofilms play an important role in the pathogenesis of various human diseases, in many other natural systems, and in the performance of man-made devices that operate in those systems. Controlling biofilm growth requires an improved understanding of biofilm development, which in turn requires novel methods of chemically characterizing biofilms in situ [1]. Signaling species, many metabolites, antimicrobial agents, and substrate degradation products are molecular species often found within biofilms and generally fall near the $2000 \mathrm{Da}$ size range. Laser desorption postionization mass spectrometry (LDPI MS) and long period x-ray standing wave fluorescence (XSW) are described for the detection of such intermediate sized molecular analytes within intact microbial biofilms, while preserving information on their spatial distribution. Chemical derivatization is used in both LDPI MS and XSW to permit selective detection of an analyte in situ.

LDPI MS involves desorption of neutrals by a pulse from one laser followed by postionization with a pulse from a second laser, which here is derived from a fluorine laser $(157 \mathrm{~nm}$ or $7.9 \mathrm{eV})$. Desorption occurs in this instrument by focusing a nitrogen laser $(337 \mathrm{~nm})$ to a spot a few micrometers in diameter using a Schwarzschild objective, resulting in ejection of mostly neutral species from the surface [2]. Selectivity is achieved with postionization by derivatizing a molecular analyte with the appropriate chemical tag to lower the ionization potential of the analyte below the $7.9 \mathrm{eV}$ photon energy. Anthracene and various other polycyclic aromatic hydrocarbons have been demonstrated as effective chemical tags for 7.9 eV LDPI MS [3,4].

Wild type Bacillus subtilis 168 biofilms were grown for several days in a Petri dish with LB media which was then changed to minimal media consistent with starvation conditions [5]. The biofilms were dried, treated with an aromatic derivatizing agent that binds to primary amine groups, then transferred into vacuum for MS analysis. A variety of amino acids and polypeptides were detected in this fashion by $7.9 \mathrm{eV}$ LDPI MS. One of these species was the ERGMT pentapeptide know to play a signaling role in Bacillus subtilis biofilms. Spiking the biofilm with synthetic ERGMT pentapeptide confirmed these results, as did traditional extraction and high resolution electrospray ionization mass spectrometric analysis. Various control experiments were performed to demonstrate the validity of the analytical method. These results compare favorably with those obtained by intact biofilm analysis using matrix assisted laser desorption ionization (MALDI) mass spectrometry. 
Fundamental constraints associated with sample preparation for analyses in vacuum and visible light spatial resolution prevent imaging LDPI MS during dynamic measurements and at spatial resolutions below a micrometers, respectively. However, XSW can be used to determine the spatial resolution of a molecular species in the condensed phase with $\sim 1 \mathrm{~nm}$ resolution. XSW was previously used to probe the conformation of a bromine labeled-polyethylene glycol-peptide construct (Br-PEG-peptide) adsorbed at the liquid-solid interface of a modified polystyrene surface [6].

Bacillus subtilis biofilms were grown for several days (see above), then transferred to either BrPEG-peptide or Br-tyrosine coated polystyrene and cultured for $\sim 3$ hours. XSW measurements of the $\mathrm{Br}$ distribution were then performed as previously described [6] except that the ChemMatCARS beamline was utilized at the Advanced Photon Source. The Br distribution was determined by XSW as the Br-tagged analyte spread into the biofilm. These results indicated that the Br-PEG-peptide layer integrity was largely maintained while the Br-tyrosine layer mostly diffused into the biofilm. However, the broadening of the Br-PEG-peptide layer distribution indicated that even this relatively strongly adsorbed layer was beginning to diffuse into or be catabolyzed by the biofilm with the few hour timescale of these experiments. Fluorescence background signal from the biofilm/polystyrene interface without added $\mathrm{Br}$ was low, indicating that the $\mathrm{Br}$ tag atom could be used to monitor less than a single layer of tagged adsorbate at the interface.

LDPI MS and XSW are described for bacterial biofilm analyses. Both methods employ chemical derivatization with either a vacuum ultraviolet postionizable or x-ray fluorescencing species to enhance the chemically selectivity of the analyte within an intact, complex mixture (the biofilm). These strategies will find wide application in studies of drug transport and metabolite detection in cells and tissue; in vitro and in vivo degradation of biomaterials and tissue engineered constructs; small molecule combinatorial arrays; and other problems [7].

\section{References}

[1] M. Ghannoum et al., Microbial Biofilms, ASM Press, Washington, D.C., 2004.

[2] I.V. Veryovkin et al., Nucl. Instr. Meth. Phys. Res. A 519 (2004) 345.

[3] P.D. Edirisinghe et al., Anal. Chem. 76 (2004) 4267.

[4] P.D. Edirisinghe et al., Appl. Surf. Sci. (2006) in press.

[5] R. Kolter, J. Bacter. 186 (2004) 3970.

[6] C.A. Crot et al., Lang. 21 (2005) 7899.

[7] This work is supported by the U.S. Department of Energy, BES-Materials Science, under Contract W-31-109-ENG-38. PDE is supported by subcontract 4J-00161-0008A from Argonne National Laboratory. 